COX2 expression rate for all 47 tumors was $46.1 \%$. A significant negative correlation was found between the pretreatment COX2 expression rate and the $\mathrm{Al} 1$ week after the initiation of radiotherapy (coefficient of correlation $r=-0.52 ; P=0.0002$ ). The Al of COX2-positive tumors during radiotherapy was $1.6 \%$, versus $2.5 \%$ for COX2-negative tumors $(P=0.0002)$. Patients with COX2-positive tumors had a lower complete response rate than patients with COX2-negative tumors (59\% vs $80 \%$ ) but this difference was not significant. When locoregional recurrence was examined, a trend towards improved locoregional control for COX2-negative tumors was identified $(P=0.06)$.

While Ishikawa et al. highlight the need for long-term data to confirm that COX2 expression inhibits radiation-induced apoptosis, they suggest that the outcome of patients with COX2-positive cervical cancer could be improved by the use of a COX2 inhibitor in combination with radiotherapy.

Original article Ishikawa $\mathrm{H}$ et al. (2006) Cyclooxygenase-2 impairs treatment effects of radiotherapy for cervical cancer by inhibition of radiation-induced apoptosis. Int $J$ Radiat Oncol Biol Phys 66: 1347-1355

\section{Preoperative chemotherapy for ovarian cancer has a negative impact}

Primary cytoreductive surgery that achieves optimal debulking of the tumor provides the best chance of survival for patients with advanced ovarian cancer; however, the impact on patient survival of suboptimal surgery with large residual margins is still unclear. Many centers have, therefore, adopted the practice of neoadjuvant platinum-based chemotherapy before cytoreductive surgery, to reduce the extent of disease and to spare patients a potentially ineffective surgical procedure. To assess the effectiveness of this strategy, Bristow and Chi have conducted a meta-analysis of studies of patients with stage III-IV ovarian cancer who were treated with neoadjuvant chemotherapy before surgery.

In all, 22 cohorts including 835 patients were analyzed. Although interval surgery that achieved maximal cytoreduction (reached in $65 \%$ of patients) was positively associated with survival, each incremental preoperative chemotherapy cycle was associated with a 4.1-month decrease in median survival time. Of the other parameters analyzed, taxane use and increasing year of study publication had positive associations with survival.

The authors conclude that preoperative neoadjuvant chemotherapy was associated with inferior patient survival compared with primary cytoreduction, and that research is required to establish criteria to distinguish patients unsuitable for primary surgery from those with resectable disease. They state that in patients undergoing neoadjuvant chemotherapy, cytoreductive surgery should be performed as early in the treatment program as possible.

Original article Bristow RE and Chi DS (2006) Platinumbased neoadjuvant chemotherapy and interval surgical cytoreduction for advanced ovarian cancer: a meta-analysis. Gynecol Oncol 103: 1070-1076

\section{TIMP-1 level identifies nonresponders to chemotherapy in metastatic breast cancer}

Cytotoxic chemotherapy is a routine treatment for metastatic breast cancer; however, many patients undergoing this treatment receive no benefit and risk unnecessary adverse effects. Noting a lack of markers for resistance to chemotherapy, Schrohl et al. investigated the relationship between expression of tissue inhibitor of metalloproteinase-1 (TIMP-1) and sensitivity to apoptosis-inducing chemotherapeutic drugs.

TIMP-1 is a known inhibitor of apoptosis, and high TIMP-1 mRNA and protein levels have been linked to poor prognosis for patients with metastatic breast cancer. This study showed that in 173 patients with this disease, log-transformed tumor expression of TIMP-1 was a significant predictor of lack of response to CMF (cyclophosphamide/methotrexate/ 5-fluorouracil; $n=94$ ) or anthracycline-based $(n=79)$ chemotherapies (odds ratio 2.0; $P=0.01)$. Importantly, the receiver operating characteristic curve showed a sensitivity of approximately $17 \%$ at a specificity of $100 \%$, indicating that TIMP-1 levels could be used to accurately identify $17 \%$ of the patients who would not respond to common chemotherapy regimens, without misclassifying any responders. Evaluation of further large, independent patient groups is needed to confirm 